\title{
Upgraded nomograms for the prediction of complications and survival in patients with colorectal liver metastases treated with neoadjuvant chemotherapy followed by hepatic resection
}

\author{
Qichen Chen, Rui Mao, Jianjun Zhao, Xinyu Bi, Zhiyu Li, Zhen Huang, Yefan Zhang, Jianguo Zhou, \\ Hong Zhao, Jianqiang Cai \\ Department of Hepatobiliary Surgery, National Cancer Center/National Clinical Research Center for Cancer/Cancer Hospital, Chinese Academy of \\ Medical Sciences and Peking Union Medical College, Beijing, China \\ Contributions: (I) Conception and design: J Zhou, H Zhao; (II) Administrative support: J Zhou, H Zhao, J Cai; (III) Provision of study materials or \\ patients: Q Chen; (IV) Collection and assembly of data: All authors; (V) Data analysis and interpretation: Q Chen, R Mao; (VI) Manuscript writing: \\ All authors; (VII) Final approval of manuscript: All authors. \\ Correspondence to: Jianguo Zhou; Hong Zhao. Department of Hepatobiliary Surgery, National Cancer Center/National Clinical Research \\ Center for Cancer/Cancer Hospital, Chinese Academy of Medical Sciences and Peking Union Medical College, Beijing 100021, China. \\ Email: zjg13311533776@126.com; zhaohong@cicams.ac.cn.
}

Background: To establish upgraded nomograms incorporating neoadjuvant chemotherapy (NAC)-related factors and preoperative testing markers to predict postoperative complications, progression-free survival (PFS) and overall survival (OS) in patients with colorectal liver metastases (CRLM).

Methods: Multivariate regression analyses were used to reveal independent predictors for postoperative complications, PFS and OS. Nomograms incorporating independent predictors were constructed, and discrimination and calibration were evaluated. Survival was estimated by the Kaplan-Meier method and compared using the log-rank test.

Results: A nomogram predicting postoperative complications was constructed based on preoperative serum gamma-glutamyl transpeptidase (GGT) $\geq 36 \mathrm{U} / \mathrm{L}$, major liver resection, intraoperative blood loss $\geq 300 \mathrm{~mL}$, primary site located in the right hemicolon and primary lymph node metastasis, with an area under the receiver operating characteristic curve (AUROC) of 0.750. The calibration curves and HosmerLemeshow test revealed desirable model calibration (chi-square: 4.47, $\mathrm{P}=0.88$ ). Moreover, a nomogram for the prediction of PFS was constructed based on tumour regression grade (TRG), primary lymph node metastasis, R0 resection and NAC cycles $\geq 5$, with good discrimination (C-index: $0.663 \pm 0.024)$ and calibration, and one for predicting OS was constructed based on preoperative GGT $\geq 36 \mathrm{U} / \mathrm{L}, \mathrm{NAC}$ toxicity, NAC cycles $\geq 5$, primary lymph node metastasis and R0 resection, with favourable discrimination (C-index: $0.684 \pm 0.030$ ) and calibration. Significant differences in PFS and OS were observed among patients stratified into three different risk groups $(\mathrm{P}<0.001)$ according to total scores based on the nomograms.

Conclusions: This study is the first to establish novel predictive nomograms specifically incorporating TRG, NAC toxicity and serum GGT level for the prediction of postoperative complications, PFS and OS in CRLM patients. The nomograms exhibit favourable discrimination and calibration to guide personalized CRLM management and therapy.

Keywords: Colorectal liver metastases (CRLM); nomogram; postoperative complications; survival

Submitted May 16, 2020. Accepted for publication Oct 26, 2020.

doi: 10.21037/atm-20-3973

View this article at: http://dx.doi.org/10.21037/atm-20-3973 


\section{Introduction}

Colorectal cancer is the third most common cancer and the second leading cause of cancer mortality worldwide (1). The liver is the most common site of distant metastasis for colorectal cancer, and approximately $50 \%$ of patients develop liver metastases during the course of their disease (2). Liver resection is generally regarded as the only potentially curative intervention for colorectal liver metastases (CRLM). As neoadjuvant chemotherapy (NAC) can treat micrometastases, reduce the tumour burden, and improve the rate of $\mathrm{R} 0$ resection, it is recommended for initially unresectable or resectable CRLM patients with high risk factors for recurrence $(3,4)$. Nonetheless, the effect of NAC combined with targeted therapy on the prognosis of patients with resectable CRLM remains controversial. A meta-analysis including 908 CRLM patients from 11 studies showed that NAC combined with targeted therapy significantly improved the effective response rate of tumours but did not improve overall survival (OS) (5). However, a recent retrospective analysis found that NAC combined with bevacizumab significantly improved the survival of patients with resectable CRLM (6). Although NAC followed by hepatic resection may result in some improvement in the prognosis of CRLM patients, $66-76 \%$ of patients experience recurrence after surgery $(7,8)$; moreover, the 5 -year OS rate is only $36-41 \%$ (8-10), and the incidence of postoperative complications is approximately $38-54 \%(9,10)$. In general, several factors are crucial for managing the treatment plan and improving the prognosis of CRLM patients, including the selection of CRLM patients whose benefits outweigh the risk of undergoing liver resection, the early identification of those who have a high risk of postoperative complications and the early identification of those who are more likely to experience recurrence after liver resection.

Nomograms provide a graphical representation of a predictive model and are used to generate a numerical probability of a clinical event and are thus helpful for formulating reasonable clinical strategies early in CRLM patient management. However, only a few studies $(7,8,11)$ have developed a nomogram for CRLM patients who received NAC followed by hepatic resection, and the previous nomograms are lacking in several ways. First, these nomograms did not consider the influence of NAC-related factors [e.g., tumour regression grade (TRG), NAC toxicity] or preoperative testing markers [e.g., gamma-glutamyl transpeptidase (GGT), D-dimer] on prognosis. TRG is a key factor used for evaluating the efficacy of NAC; it has been reported to be significantly associated with clinical outcomes in CRLM patients and is considered an indispensable factor for predicting CRLM patient survival $(9,12)$. Preoperative testing markers (GGT, D-dimer, etc.) have recently been found to be novel independent factors for the prediction of survival and postoperative complications in CRLM patients (13). It is hypothesized that the incorporation of these factors into existing models would enhance the predictive power. Second, given the high incidence of postoperative complications and subsequent adverse impact on the quality of life of patients, it is important to construct a nomogram for the prediction of postoperative complications in CRLM patients who receive NAC followed by resection. As there is a lack of a relevant nomograms and considering the deficits in current models, this study was designed to develop and upgrade nomograms for the prediction of postoperative complications and survival in CRLM patients in an attempt to address the above deficiencies. We present the following article in accordance with the STROBE reporting checklist (available at http://dx.doi.org/10.21037/atm-20-3973).

\section{Methods}

\section{Patients}

The present study enrolled 169 CRLM patients who underwent NAC followed by hepatic resection between February 2010 and February 2018 at Cancer Hospital, Chinese Academy of Medical Sciences. The study conformed to the provisions of the Declaration of Helsinki (as revised in 2013). Ethical approval was obtained from our hospital (approval ID: NCC2019C-016). All participants gave informed consent before taking part in this study. The inclusion criteria were as follows: (I) pathologically proven colorectal adenocarcinoma liver metastases and (II) treatment including NAC followed by hepatic resection for curative intent. The exclusion criteria were as follows: (I) treatment with neoadjuvant radiotherapy; (II) other malignancies; and (III) loss to follow-up or incomplete clinical data. Chronic medical diseases, including diabetes, hypertension, and cardiac disease, were defined as comorbidities. Preoperative serum GGT levels (normal range, $0-55 \mathrm{U} / \mathrm{L}$ ) were measured by using an enzyme kinetic assay within 1 week before surgery.

\section{NAC and surgical treatment}

NAC was recommended for unresectable CRLM patients 
and resectable CRLM patients with high risk factors for recurrence (e.g., primary lymph node metastasis, synchronous liver metastasis, multiple metastases) (4). The NAC regimen included oxaliplatin- or irinotecan-based regimens, such as FOLFOX (5-fluorouracil/leucovorin/ oxaliplatin), XELOX (capecitabine/oxaliplatin) or FOLFIRI (5-fluorouracil/leucovorin/irinotecan). Targeted therapy included bevacizumab and cetuximab. NAC toxicities, including haematologic toxicity, gastrointestinal toxicity, and neurotoxicity, were graded using NCI-CTCAE (version 4.0). Clinical tumour response was assessed by imaging every two NAC cycles according to the RECIST. After surgery, pathological response was evaluated according to TRG, and TRG 1-3 was defined as a pathological response to NAC (14).

During NAC, the possibility for surgery was assessed by MDT, as previously described (15). Patients usually underwent liver resection within 4-6 weeks after the completion of NAC. Liver resection was defined as major or minor liver resection. Major liver resection was defined as resection of more than two segments. $\mathrm{R} 0$ resection was defined as a distance from the tumour margin to the transection line greater than $1 \mathrm{~mm}$. Postoperative complications were graded according to the Clavien-Dindo system, and major complications were defined as grade III or IV complications (16). If patients experienced multiple postoperative complications, the highest grade was used.

\section{Follow-up and outcomes}

Patients were followed up at regular intervals after surgery. The first follow-up was 1 month after surgery, with subsequent follow-ups every 3 months for 2 years, every 6 months between years $2-5$, and every 1 year thereafter. Progression or recurrence was detected by the carcinoembryonic antigen (CEA) level and imaging. OS was defined as the interval from the date of resection to death or the last follow-up. Progression-free survival (PFS) was defined as the interval from the date of resection to progression or the last follow-up.

\section{Statistical analysis}

The Mann-Whitney $U$ test was performed to analyse continuous variables, and chi-square or Fisher's exact tests were used to analyse categorical variables. OS and PFS were calculated by the Kaplan-Meier method and compared with the log-rank test. All predictors with $\mathrm{P}<0.10$ in the univariate analysis were retained in the multivariate models.
Multivariate analysis of relationships between characteristics and complications was performed using a logistic regression analysis model; the Cox regression model was employed for the multivariate analysis of survival. $\mathrm{P}<0.05$ was considered statistically significant. Independent predictors were retained for the construction of a nomogram. The $\mathrm{C}$-index and area under the receiver operating characteristic curve (AUROC) were applied to investigate model discrimination. Calibration plots were generated, and the Hosmer-Lemeshow chi-square test was conducted to assess model calibration. Decision curve analysis (DCA) was applied to investigate clinical usefulness. $\mathrm{X}$-tile analysis was implemented to determine the optimal segmentation threshold for survival curve risk stratification. SPSS software version 22 (Armonk NV, USA) and R software (http://www. r-project.org) were used to perform the statistical analyses.

\section{Results}

\section{Clinicopathological characteristics}

One hundred sixty-nine patients, including 60 females and 109 males, were included in this study, with a median age of 55 (IQR, 49.5-62.0) years. A preoperative serum GGT level of $\geq 36 \mathrm{U} / \mathrm{L}$ was recorded for 83 patients. Primary tumours located in the right hemicolon were found in 23 patients (13.6\%). Lymph node metastasis of the primary tumour was observed in $71.6 \%$ of the patients, and bilobar distribution of liver metastases was found in 88 (52.1\%). The median diameter of the largest liver metastasis was 3.0 (IQR, 2.0-4.0) cm, and in 86 patients, the largest liver metastasis was $\geq 3 \mathrm{~cm}$ in diameter. Moreover, $71.6 \%$ of patients had more than one liver metastasis, with a median of 3 lesions (IQR, 1.0-4.5). One hundred forty-two patients $(84.0 \%)$ underwent major liver resection. The median operation time was 345 (IQR, 255-425) min, with median intraoperative blood loss of 300 (IQR, 150-500) mL. In study, 21 CRLM patients had extrahepatic metastases.

One hundred and fourteen patients $(67.5 \%)$ received an oxaliplatin-based NAC regimen; 58 patients (34.3\%) underwent targeted therapy (bevacizumab: 30 patients; cetuximab: 27 patients; bevacizumab + cetuximab: 1 patient). In total, $54.4 \%$ of patients received more than 5 NAC cycles. NAC toxicities were observed in 149 patients (88.2\%), including 59 with haematologic toxicity (grades 1-2: 45 patients; grades 3-4: 14 patients), 120 with gastrointestinal toxicity (grades 1-2: 115 patients; grades 3-4: 5 patients), 21 with skin and mucous membrane toxicity (grades $1-2$ : 
Table 1 Demographic and clinical characteristics of study patients

\begin{tabular}{|c|c|}
\hline Variety & All patients $(n=169)(\%)$ \\
\hline Age $\geq 60$ years & $60(35.5)$ \\
\hline Male & $109(64.5)$ \\
\hline $\mathrm{BMI} \geq 24 \mathrm{~kg} / \mathrm{m}^{2}$ & $89(52.7)$ \\
\hline Comorbidity & $73(43.2)$ \\
\hline ASA score 3-4 & $21(12.4)$ \\
\hline Preoperative CEA $\geq 10$ ng/mL & $73(43.2)$ \\
\hline Preoperative GGT $\geq 36 \mathrm{U} / \mathrm{L}$ & $83(49.1)$ \\
\hline Preoperative D-dimer $\geq 0.49$ mg/L & $85(50.3)$ \\
\hline Primary site in colon & $92(54.4)$ \\
\hline Right hemicolon & $23(13.6)$ \\
\hline Poor differentiation & $42(24.9)$ \\
\hline T3-T4 stage & $157(92.9)$ \\
\hline Primary lymph node metastasis & $121(71.6)$ \\
\hline Synchronous metastasis & $146(86.4)$ \\
\hline Diameter of metastases $\geq 3 \mathrm{~cm}$ & $86(50.9)$ \\
\hline Bilobar liver distribution & $88(52.1)$ \\
\hline Extrahepatic metastases & $21(12.4)$ \\
\hline KRAS mutation ${ }^{a}$ & $34(20.1)$ \\
\hline \multicolumn{2}{|l|}{ Surgery details } \\
\hline Operation time(min), media (IQR) & $345(255-425)$ \\
\hline Blood loss (mL), median (IQR) & $300(150-500)$ \\
\hline Major liver resection & $142(84.0)$ \\
\hline Concomitant RFA & $35(20.7)$ \\
\hline Heterochronous resection & $46(27.2)$ \\
\hline $\mathrm{R} 0$ resection & $107(63.3)$ \\
\hline Postoperative complications & $90(53.3)$ \\
\hline \multicolumn{2}{|l|}{ Neoadjuvant chemotherapy details } \\
\hline Oxaliplatin based regimen & $114(67.5)$ \\
\hline Cycles $\geq 5$ & $92(54.4)$ \\
\hline Targeted therapy & $58(34.3)$ \\
\hline Second-line chemotherapy & $30(17.8)$ \\
\hline NAC toxicities & $149(88.2)$ \\
\hline Pathological response & $66(39.1)$ \\
\hline Postoperative chemotherapy & $105(62.1)$ \\
\hline Multiple metastases & $121(71.6)$ \\
\hline
\end{tabular}

${ }^{\mathrm{a}}$, the status of KRAS mutation was reached in 114 patients. BMI, body mass index; ASA, American Society of Anesthesiologists physical status classification; CEA, carcinoembryonic antigen; GGT, gamma-glutamyltranspeptidase; IQR, interquartile range; RFA, radiofrequency ablation; NAC, neoadjuvant chemotherapy.
18 patients; grades 3-4: 3 patients) and 15 with neurotoxicity (grades 1-2). A favourable pathological response (TRG 1-3) was observed in 66 patients (39.1\%). The demographic and clinical characteristics of the patients are listed in Table 1.

\section{Predictors for postoperative complications}

In this study, $53.3 \%$ of patients experienced postoperative complications (general complications: 55 patients; surgeryrelated complications: 59 patients), including 36 major complications and 54 minor complications. In the univariate analysis (Table 2), preoperative serum GGT $\geq 36 \mathrm{U} / \mathrm{L}$ $(\mathrm{P}=0.001)$, major liver resection $(\mathrm{P}=0.010)$, and intraoperative blood loss $\geq 300 \mathrm{~mL}$ ( $\mathrm{P}=0.009$ ) were significantly associated with postoperative complications. A tendency towards postoperative complications was also detected for patients whose primary tumour was located in the right hemicolon, those with primary lymph node metastasis and those with a diameter of the largest liver metastasis $\geq 3 \mathrm{~cm}(\mathrm{P}<0.1)$.

All of the abovementioned predictors $(\mathrm{P}<0.1)$ were included in the multivariate analysis, and preoperative serum GGT $\geq 36 \mathrm{U} / \mathrm{L}$ [odds ratio $(\mathrm{OR})=2.663,95 \%$ confidence interval (CI): $1.361-5.210, \mathrm{P}=0.004]$, major liver resection $(\mathrm{OR}=2.802,95 \%$ CI: 1.056-7.440, $\mathrm{P}=0.039)$, intraoperative blood loss $\geq 300 \mathrm{~mL}$ (OR $=2.731,95 \%$ CI: $1.370-5.446$, $\mathrm{P}=0.004)$, right hemicolon $(\mathrm{OR}=3.677$, 95\% CI: 1.220 $11.082, \mathrm{P}=0.021)$ and primary lymph node metastasis $(\mathrm{OR}$ $=2.460,95 \%$ CI: $1.138-5.318, \mathrm{P}=0.022$ ) were independently associated with the presence of postoperative complications (Table 2).

\section{Construction of a nomogram for the prediction of postoperative complications}

A nomogram with five independent predictors from the multivariate analysis was developed (Figure 1). These factors were assigned specific scores as follows: preoperative serum GGT $\geq 36$ U/L, 75; major liver resection, 79; intraoperative blood loss $\geq 300 \mathrm{~mL}, 77$; right hemicolon, 100 ; and primary lymph node metastasis, 69. Total risk scores for each patient were calculated based on the nomogram, with the total points ranging from 0 to 400 . The cut-off value was set at 176 according to the ROC curve, with a sensitivity of 0.811 and a specificity of 0.620 . The performance of the model in predicting postoperative complications was acceptable, with an AUROC of 0.750 (95\% CI: 0.676-0.824) (Figure 2A), and calibration curves and the Hosmer-Lemeshow test revealed desirable model calibration (chi-square: 4.47, 
Table 2 Prognostic factors for complications in CRLM patients after liver resection

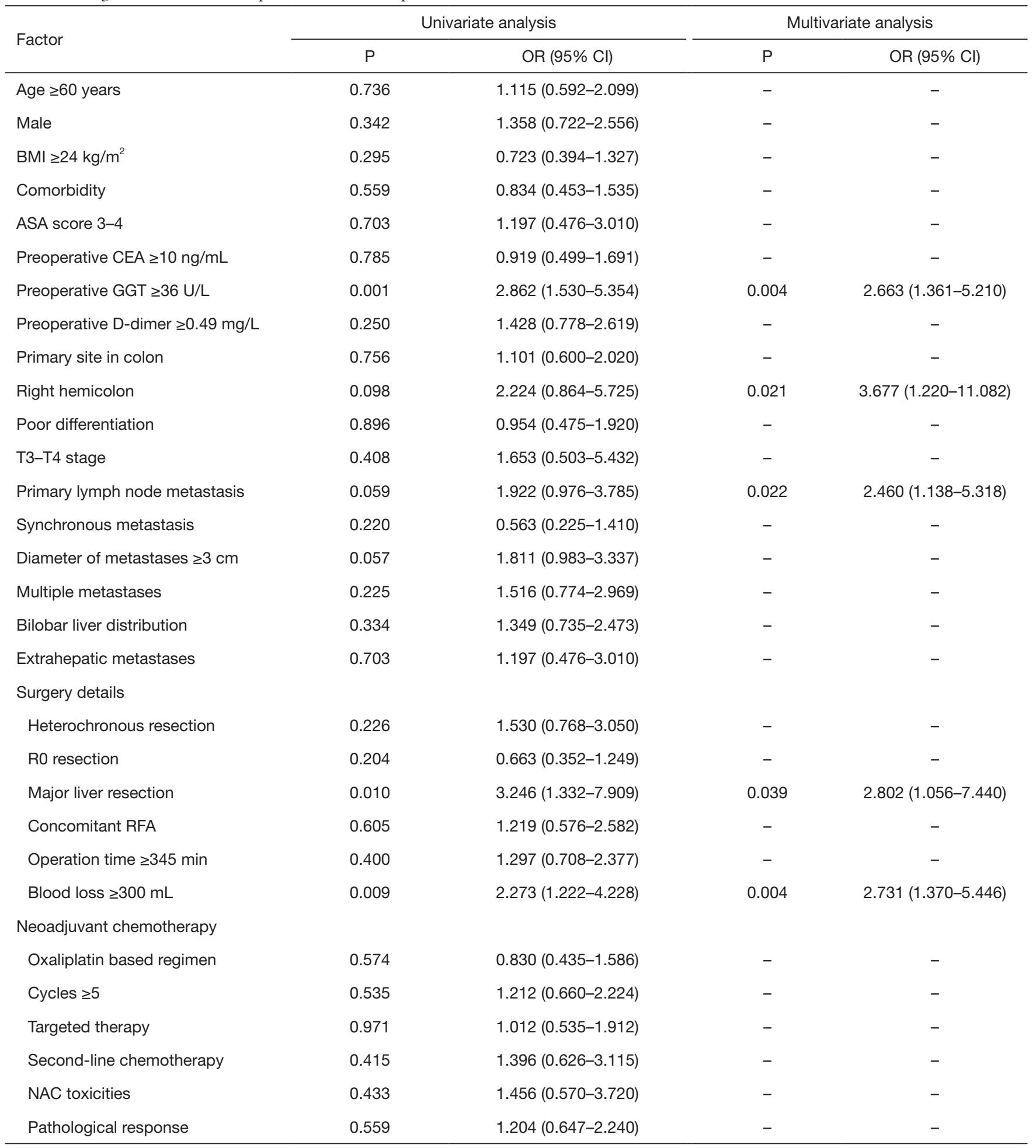

BMI, body mass index; OR, odds ratio; Cl, confidence interval; CRLM, colorectal liver metastases; ASA, American Society of Anesthesiologists physical status classification; CEA, carcinoembryonic antigen; GGT, gamma-glutamyltranspeptidase; RFA, radiofrequency ablation; NAC, neoadjuvant chemotherapy. 


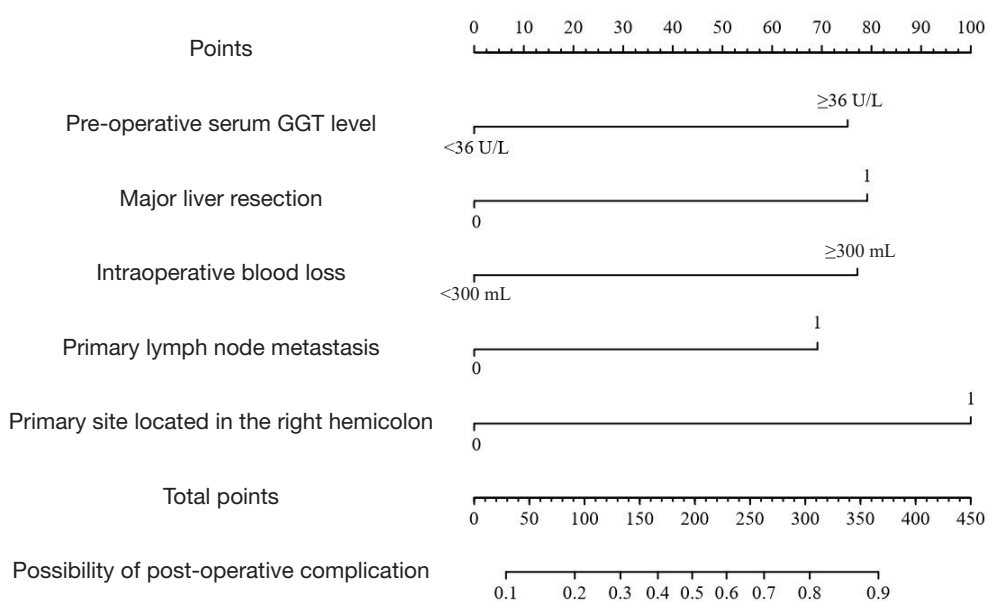

Figure 1 Nomogram predicting the probability of post-operative complications in CRLM patients. Total points were calculated by adding each variable on the point scale, and the results indicate the probability of post-operative complications according to the bottom scales. CRLM, colorectal liver metastases; GGT, gamma-glutamyl transpeptidase.
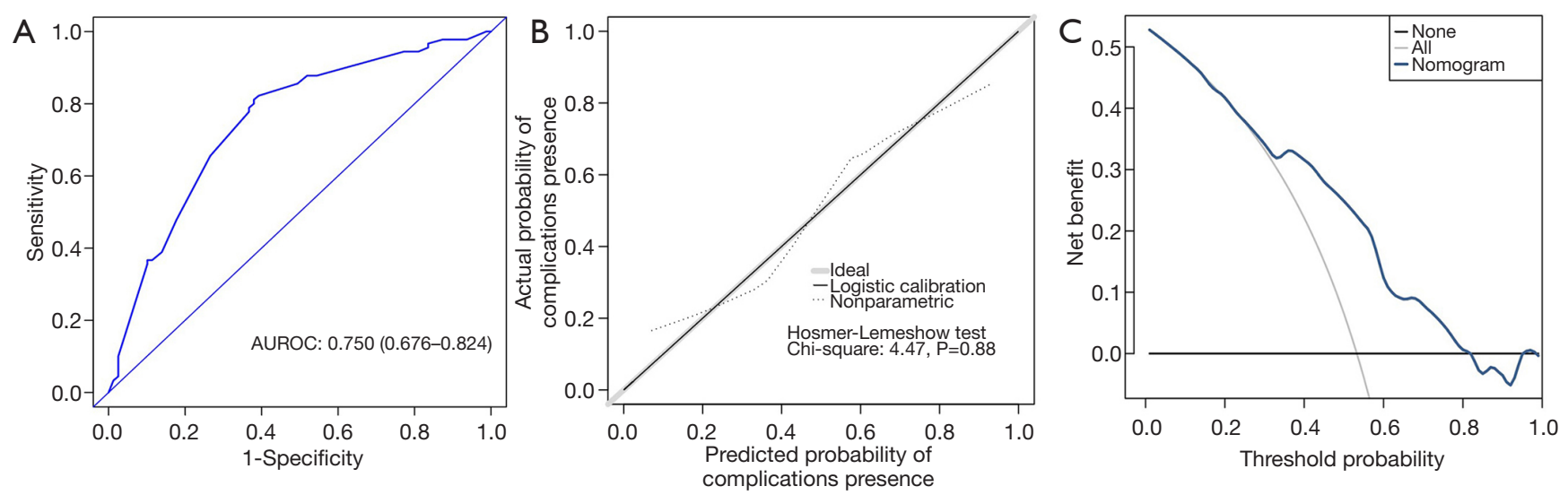

Figure 2 ROC curves, calibration curves, decision curve analysis for the nomogram in the prediction of post-operative complications. (A) ROC curves of the nomogram in the prediction of post-operative complications; (B) calibration curves for predicting complications; (C) decision curve analysis (DCA) for the nomogram. AUROC, area under the receiver operating characteristic curve; ROC, receiver operating characteristic.

$\mathrm{P}=0.88$ ) (Figure 2B). Moreover, DCA demonstrated that using this nomogram to predict postoperative complications was probably beneficial (Figure 2C).

\section{Prognostic factors for PFS}

One hundred forty-four patients (85.2\%) experienced tumour progression. The median PFS duration was 7.9 (IQR, 5.9-10.0) months, and the 1- and 3-year PFS rates were $34.9 \%$ and $15.0 \%$, respectively. In the univariable analysis, stages $\mathrm{T} 3-\mathrm{T} 4$, primary lymph node metastasis, multiple metastases, bilobar liver distribution, non-R0 resection, NAC cycles $\geq 5$, second-line chemotherapy, targeted therapy and non-pathological response were related to decreased PFS $(\mathrm{P}<0.05)$. In addition, four independent prognostic factors for PFS were identified in the multivariable analysis: primary lymph node metastasis [hazard ratio $(\mathrm{HR})=1.722$, 95\% CI: 1.162-2.551, $\mathrm{P}=0.007$ ], R0 resection ( $\mathrm{HR}=0.585,95 \%$ CI: $0.415-0.823, \mathrm{P}=0.002$ ), NAC cycles $\geq 5(\mathrm{HR}=1.487,95 \% \mathrm{CI}: 1.066-2.074$, $\mathrm{P}=0.019)$ and pathological response $(\mathrm{HR}=0.688,95 \% \mathrm{CI}$ : 0.482-0.981, $\mathrm{P}=0.039$ ) (Table 3). 
Table 3 Prognostic factors for PFS in CRLM patients after liver resection

\begin{tabular}{|c|c|c|c|c|}
\hline Factor & \multicolumn{2}{|c|}{ Univariate analysis } & \multicolumn{2}{|c|}{ Multivariate analysis } \\
\hline Age $\geq 60$ years & 0.139 & $0.767(0.540-1.090)$ & - & - \\
\hline Male & 0.583 & $1.101(0.781-1.552)$ & - & - \\
\hline $\mathrm{BMI} \geq 24 \mathrm{~kg} / \mathrm{m}^{2}$ & 0.328 & $1.177(0.849-1.634)$ & - & - \\
\hline ASA score 3-4 & 0.122 & $1.467(0.903-2.384)$ & - & - \\
\hline Preoperative CEA $\geq 10 \mathrm{ng} / \mathrm{mL}$ & 0.585 & $1.097(0.787-1.527)$ & - & - \\
\hline Preoperative GGT $\geq 36 \mathrm{U} / \mathrm{L}$ & 0.066 & $1.360(0.980-1.889)$ & - & - \\
\hline Preoperative D-dimer $\geq 0.49 \mathrm{mg} / \mathrm{L}$ & 0.694 & $1.068(0.770-1.481)$ & - & - \\
\hline T3-T4 stage & 0.041 & $2.216(1.035-4.748)$ & - & - \\
\hline Primary lymph node metastasis & 0.002 & 1.865 (1.266-2.748) & 0.007 & $1.722(1.162-2.551)$ \\
\hline Synchronous metastasis & 0.096 & $1.522(0.928-2.496)$ & - & - \\
\hline Diameter of metastases $\geq 3 \mathrm{~cm}$ & 0.977 & $0.995(0.717-1.381)$ & - & - \\
\hline Multiple metastases & 0.001 & $1.893(1.289-2.781)$ & - & - \\
\hline Bilobar liver distribution & 0.001 & 1.748 (1.254-2.437) & - & - \\
\hline Extrahepatic metastases & 0.886 & $0.964(0.588-1.581)$ & - & - \\
\hline Operation time $\geq 345 \mathrm{~min}$ & 0.057 & $1.376(0.991-1.909)$ & - & - \\
\hline Blood loss $\geq 300 \mathrm{~mL}$ & 0.876 & $1.026(0.738-1.427)$ & - & - \\
\hline Postoperative complications & 0.171 & 1.257 (0.906-1.745) & - & - \\
\hline \multicolumn{5}{|l|}{ Neoadjuvant chemotherapy } \\
\hline Oxaliplatin based regimen & 0.039 & $0.695(0.492-0.982)$ & - & - \\
\hline Cycles $\geq 5$ & 0.010 & $1.543(1.107-2.151)$ & 0.019 & $1.487(1.066-2.074)$ \\
\hline Targeted therapy & 0.004 & 1.653 (1.177-2.322) & - & - \\
\hline Second-line chemotherapy & 0.033 & $1.570(1.037-2.376)$ & - & - \\
\hline NAC toxicities & 0.763 & $0.925(0.557-1.536)$ & - & - \\
\hline Postoperative chemotherapy & 0.157 & $0.785(0.561-1.098)$ & - & - \\
\hline Pathological response & 0.004 & $0.602(0.426-0.852)$ & 0.039 & $0.688(0.482-0.981)$ \\
\hline
\end{tabular}

PFS, progression-free survival; CRLM, colorectal liver metastases; HR, hazard ratio; Cl, confidence interval; BMI, body mass index; ASA, American Society of Anesthesiologists physical status classification; CEA, carcinoembryonic antigen; GGT, gammaglutamyltranspeptidase; RFA, radiofrequency ablation. 

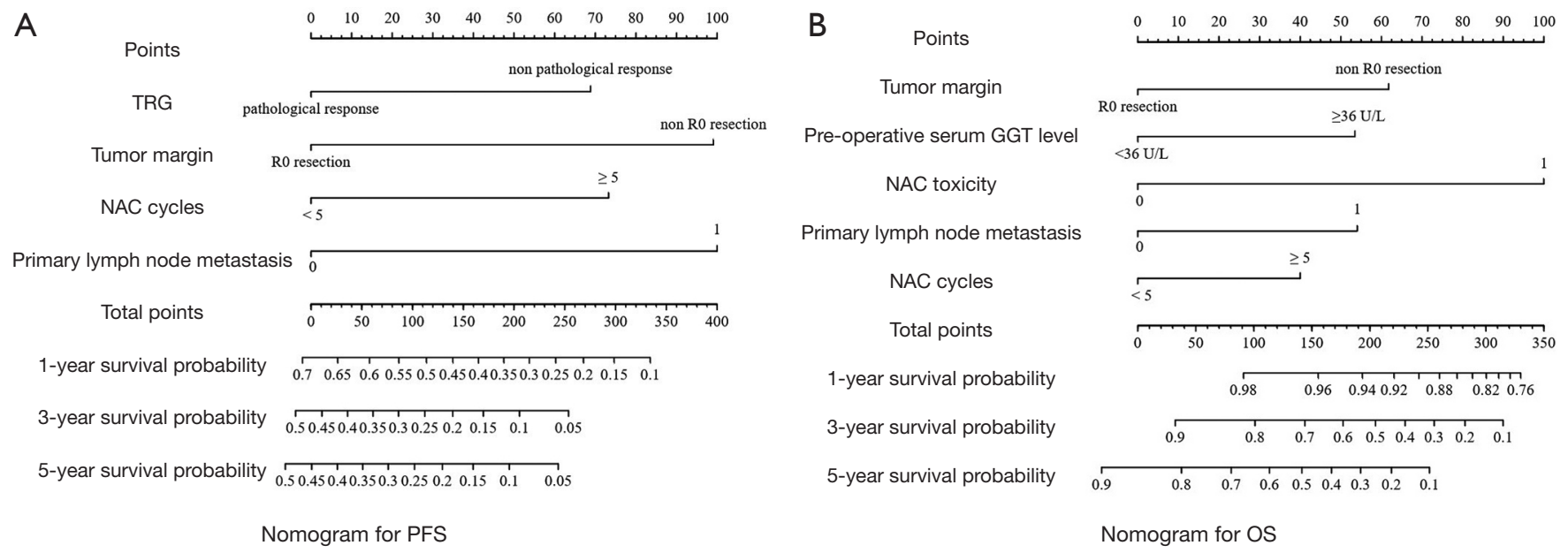

Figure 3 Nomograms for survival. (A) Nomogram for PFS; (B) nomogram for OS. The sum of the scores for each variable is plotted on the total points axis; the estimated probabilities of PFS or OS at 1,3 and 5 years were obtained by drawing a line perpendicularly from the plotted total points axis straight to the survival axis. TRG, tumour regression grade; NAC, neoadjuvant chemotherapy; GGT, gammaglutamyl transpeptidase; PFS, progression-free survival; OS, overall survival.

\section{Construction of a nomogram for PFS prediction}

A prognostic nomogram for PFS with point scales for the above four independent prognostic factors was constructed (Figure 3). These factors were assigned specific scores as follows: primary lymph node metastasis, 100; non-R0 resection, 99; NAC cycles $\geq 5,73$; and non-pathological response, 69. The C-statistic for PFS prediction was $0.663 \pm 0.024$. Furthermore, a calibration plot for the probability of PFS at 1, 3 and 5 years (Figure 4) demonstrated good calibration between the predictions by the nomogram and the actual observations.

We also calculated total risk scores for each patient based on the nomogram, and the total points ranged from 0 to 341 . The optimal segmentation threshold for dividing patients into three subgroups (high-risk: 243-341, middlerisk: $173-242$ and low-risk: $0-172$ groups) according to the total risk scores was determined by $\mathrm{X}$-tile analysis. As shown in Figure 5, the high-risk group was associated with substantially worse PFS than both the middle-risk group $(\mathrm{P}=0.001 ; \mathrm{mPFS}: 4.0$ vs. 7.7) and the low-risk group $(\mathrm{P}<0.001$; mPFS: 4.0 vs. 11.2). Additionally, the middle-risk group exhibited significantly worse PFS than the low-risk group ( $\mathrm{P}=0.020$; mPFS: 7.7 vs. 11.2).

\section{Prognostic factors for OS}

In this study, 96 (56.8\%) of the enrolled patients died. The median OS duration was 41.0 (IQR, 35.2-46.8) months, and the $1-$ and 3 -year OS rates were $92.3 \%$ and $51.0 \%$, respectively. In the univariable analysis, preoperative GGT $\geq 36 \mathrm{U} / \mathrm{L}$, primary lymph node metastasis, bilobar liver distribution, non-R0 resection, postoperative complications, NAC cycles $\geq 5$, NAC toxicities and non-postoperative chemotherapy were related to decreased $\mathrm{OS}(\mathrm{P}<0.05)$. Five independent prognostic factors for OS were identified in the multivariable analysis: preoperative GGT $\geq 36 \mathrm{U} / \mathrm{L}$ (HR $=1.792,95 \%$ CI: 1.181-2.719, $\mathrm{P}=0.006)$, primary lymph node metastasis (HR $=1.799,95 \%$ CI: $1.102-2.937$, $\mathrm{P}=0.019)$, R0 resection (HR $=0.510,95 \%$ CI: $0.337-0.772$, $\mathrm{P}=0.001)$, NAC cycles $\geq 5$ ( $\mathrm{HR}=1.544,95 \% \mathrm{CI}: 1.004$ 2.372, $\mathrm{P}=0.048)$ and NAC toxicities (HR $=2.973,95 \% \mathrm{CI}$ : 1.078-8.200, $\mathrm{P}=0.035$ ) (Table 4).

\section{Construction of a nomogram for OS prediction}

A prognostic nomogram for OS after resection with point scales for the above five independent prognostic factors was produced (Figure 3). These factors were assigned specific scores as follows: preoperative GGT $\geq 36 \mathrm{U} / \mathrm{L}, 53$; primary lymph node metastasis, 54; non-R0 resection, 62; NAC cycles $\geq 5,40$; and NAC toxicities, 100. The C-statistic for OS prediction was $0.684 \pm 0.030$. A calibration plot for the probability of survival at 1,3 and 5 years (Figure 4) demonstrated good calibration between the predictions by the nomogram and the actual observations. 

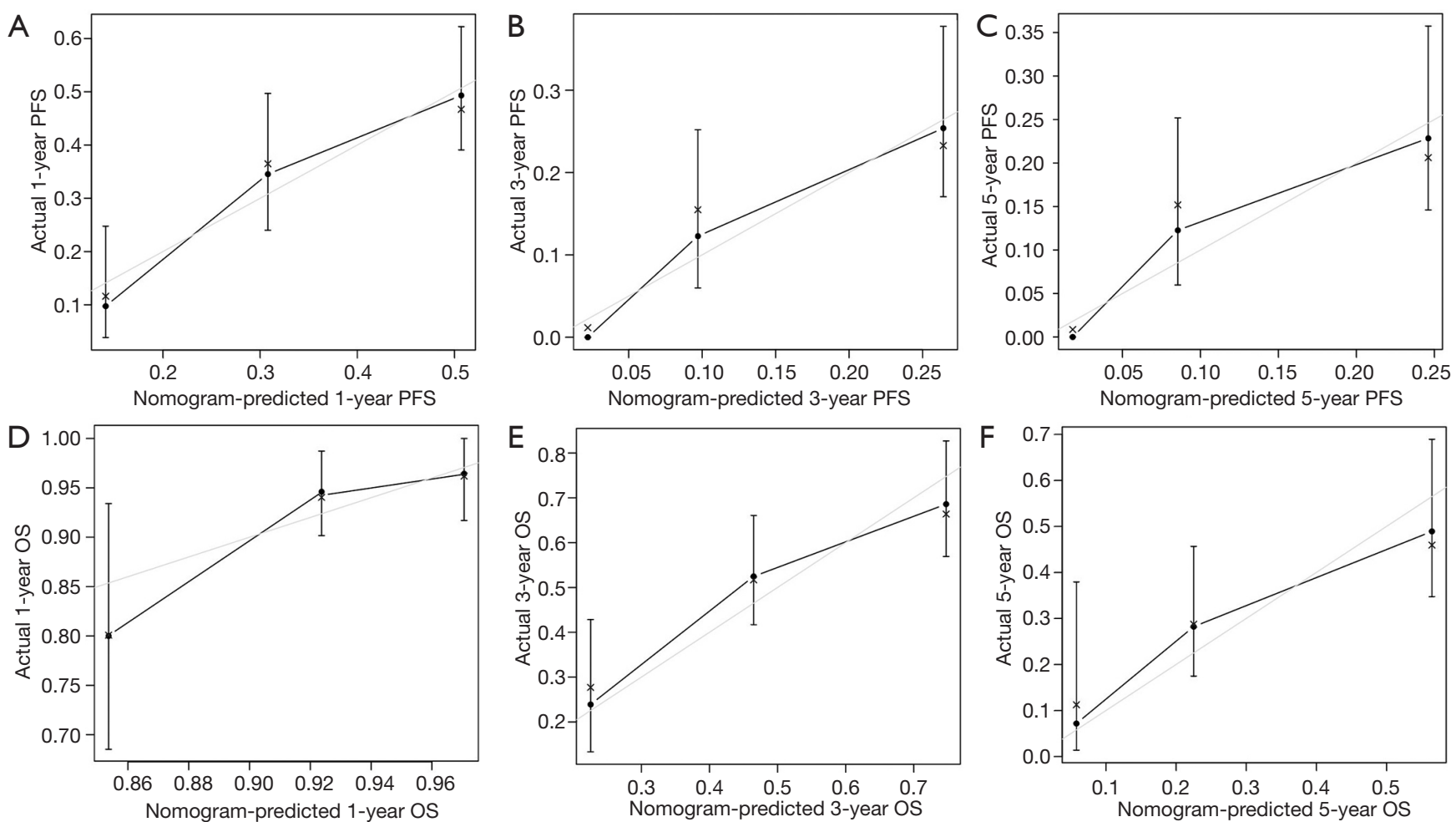

Figure 4 Calibration curves for predicting 1-year (A), 3-year (B) and 5-year (C) PFS and 1-year (D), 3-year (E) and 5-year (F) OS. Predicted survival produced by the nomogram is plotted on the $\mathrm{x}$-axis, and actual survival is plotted on the $\mathrm{y}$-axis. Dashed lines represent an identical calibration model in which the predicted PFS or OS approximate the actual PFS or OS. PFS, progression-free survival; OS, overall survival.
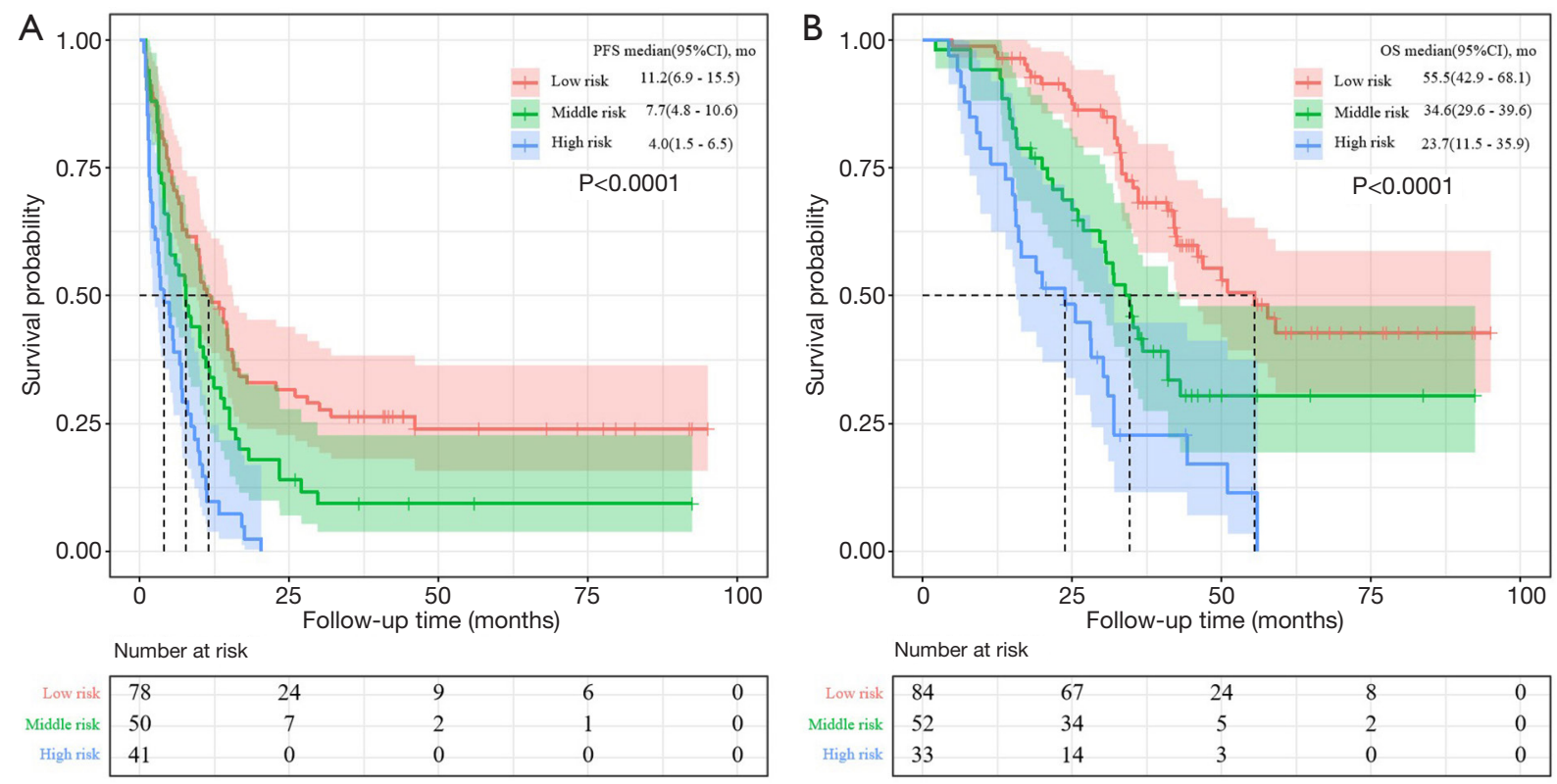

Figure 5 PFS (A) and OS (B) analysis of the three different risk groups. CI, confidence interval; PFS, progression-free survival; OS, overall survival. 
Table 4 Prognostic factors for OS in CRLM patients after liver resection

\begin{tabular}{|c|c|c|c|c|}
\hline Factor & \multicolumn{2}{|c|}{ Univariate analysis } & \multicolumn{2}{|c|}{ Multivariate analysis } \\
\hline Age $\geq 60$ years & 0.316 & $0.800(0.517-1.237)$ & - & - \\
\hline Male & 0.812 & $1.052(0.692-1.599)$ & - & - \\
\hline $\mathrm{BMI} \geq 24 \mathrm{~kg} / \mathrm{m}^{2}$ & 0.266 & $1.258(0.839-1.886)$ & - & - \\
\hline ASA score 3-4 & 0.617 & $0.857(0.467-1.571)$ & - & - \\
\hline Preoperative CEA $\geq 10 \mathrm{ng} / \mathrm{mL}$ & 0.679 & $1.089(0.727-1.632)$ & - & - \\
\hline Preoperative GGT $\geq 36 \mathrm{U} / \mathrm{L}$ & 0.004 & $1.809(1.203-2.721)$ & 0.006 & $1.792(1.181-2.719)$ \\
\hline Preoperative D-dimer $\geq 0.49 \mathrm{mg} / \mathrm{L}$ & 0.264 & $1.258(0.841-1.880)$ & - & - \\
\hline T3-T4 stage & 0.053 & $3.119(0.987-9.855)$ & - & - \\
\hline Primary lymph node metastasis & 0.020 & $1.780(1.095-2.892)$ & 0.019 & $1.799(1.102-2.937)$ \\
\hline Synchronous metastasis & 0.770 & $1.095(0.597-2.006)$ & - & - \\
\hline Diameter of metastases $\geq 3 \mathrm{~cm}$ & 0.132 & $1.362(0.911-2.038)$ & - & - \\
\hline Multiple metastases & 0.112 & $1.449(0.917-2.290)$ & - & - \\
\hline Bilobar liver distribution & 0.019 & $1.628(1.083-2.447)$ & - & - \\
\hline Extrahepatic metastases & 0.856 & $1.058(0.577-1.938)$ & - & - \\
\hline Operation time $\geq 345 \mathrm{~min}$ & 0.257 & $1.261(0.844-1.885)$ & - & - \\
\hline Blood loss $\geq 300 \mathrm{~mL}$ & 0.306 & $1.236(0.824-1.856)$ & - & - \\
\hline Postoperative complications & 0.004 & $1.814(1.205-2.729)$ & - & - \\
\hline \multicolumn{5}{|l|}{ Neoadjuvant chemotherapy } \\
\hline Oxaliplatin based regimen & 0.047 & $0.653(0.429-0.995)$ & - & - \\
\hline Cycles $\geq 5$ & 0.003 & $1.902(1.252-2.890)$ & 0.048 & $1.544(1.004-2.372)$ \\
\hline Targeted therapy & 0.054 & $1.501(0.994-2.268)$ & - & - \\
\hline Second-line chemotherapy & 0.066 & 1.605 (0.969-2.659) & - & - \\
\hline NAC toxicities & 0.024 & $3.156(1.160-8.592)$ & 0.035 & $2.973(1.078-8.200)$ \\
\hline Postoperative chemotherapy & 0.028 & $0.635(0.423-0.953)$ & - & - \\
\hline Pathological response & 0.059 & $0.667(0.438-1.015)$ & - & - \\
\hline
\end{tabular}

OS, overall survival; CRLM, colorectal liver metastases; HR, hazard ratio; Cl, confidence interval; BMI, body mass index; ASA, American Society of Anesthesiologists physical status classification; CEA, carcinoembryonic antigen; GGT, gamma-glutamyltranspeptidase; RFA, radiofrequency ablation. 
The total risk scores ranged from 0 to 309 for each patient based on the nomogram, and $\mathrm{X}$-tile analysis was conducted to determine the optimal segmentation threshold for dividing patients into three subgroups (high-risk: 256-309, middle-risk: 195-255 and low-risk: 0-194 groups) according to these total risk scores. As depicted in Figure 5, the high-risk group exhibited significantly worse OS than the middle-risk ( $\mathrm{P}=0.014$; mOS: 23.7 vs. 34.6) and low-risk $(\mathrm{P}<0.001$; mOS: 23.7 vs. 55.5) groups, and the middle-risk group was associated with significantly worse OS than the low-risk group ( $\mathrm{P}=0.001$; mOS: 34.6 vs. 55.5).

\section{Discussion}

This study successfully established upgraded nomograms by specifically considering NAC-related factors and preoperative testing markers to predict postoperative complications, PFS and OS in CRLM patients undergoing NAC followed by hepatic resection. Each factor, identified as an independent predictor by multivariable regression analysis, has a different weight in the nomogram, which should be quantified and specified when clinical strategies are determined for personalized CRLM management and therapy.

To our knowledge, this study is the first to develop a nomogram for the prediction of postoperative complications in CRLM patients. The present nomogram showed desirable performance in both discrimination $(\mathrm{ROC}=0.750)$ and calibration (chi-square: $4.47, \mathrm{P}=0.88$ ). The nomogram integrates several independent predictive factors, including preoperative test markers (serum GGT level), surgical conditions (major liver resection and intraoperative blood loss) and tumour biological characteristics (primary tumours located in the right hemicolon and primary lymph node metastasis). Previous findings indicated high serum GGT levels to be significantly related to the occurrence of postoperative major complications in CRLM patients who did not receive NAC (13), and our study further demonstrates their positive relationship with postoperative complications in CRLM patients undergoing NAC followed by hepatic resection. Overall, the extent of hepatectomy (minor liver resection or major liver resection) and intraoperative blood loss are important factors associated with trauma caused by surgery in patients $(17,18)$. As major liver resection and greater intraoperative blood loss cause serious trauma, these factors have a positive relationship with and are predictive of the occurrence of postoperative complications. It is interesting that our study is the first to reveal that primary tumours located in the right hemicolon and primary lymph node metastasis are related to postoperative complications. The possible mechanisms are as follows. Primary lymph node metastasis often is accompanied by enlarged lymph nodes closely adhering to the surrounding tissue (19), which causes great difficulty for lymphadenectomy, and lymphadenectomy of enlarged lymph nodes is often accompanied by local tissue injury and blood loss (20). Thus, the correlation between primary lymph node metastasis and the occurrence of postoperative complications is reasonable. Patients with cancer in the right hemicolon usually present with systemic symptoms (fatigue, anaemia, etc.) (21), which represent a poor condition. In addition, right hemicolon resection has a higher surgical risk than does left hemicolon resection (21-23). Therefore, a relationship between primary tumours in the right hemicolon and postoperative complications is expected.

This study also established nomograms for the prediction of OS and PFS in CRLM patients undergoing NAC followed by resection. Regarding the independent prognostic factors in the nomograms, non-R0 resection and primary lymph node metastasis were found to be associated with poor OS and PFS, which was consistent with the findings of previous studies $(7,8,24)$. Some nomograms have been developed to predict individual survival probabilities for CRLM patients receiving NAC $(7,8,11)$, but the present nomograms have some unique features. First, TRG was incorporated into the nomogram for predicting PFS. For CRLM patients receiving NAC following resection, TRG, which takes into account the levels of necrosis and fibrosis, as well as the number of viable tumour cells, is the key criterion used to evaluate the response to NAC $(9,14)$. The findings of previous studies highlight the strong association between pathological response and prolonged PFS in CRLM patients and consider TRG to be an indispensable factor for assessing CRLM patient survival $(9,12)$. Second, preoperative testing of the serum GGT level was first proven to be associated with OS in CRLM patients receiving NAC and included in the nomogram for the prediction of OS. Previous studies have validated the use of the serum GGT level as a predictor of survival in hepatocellular carcinoma, oesophageal squamous cell carcinoma, and renal cell carcinoma patients as well as CRLM patients not treated with NAC (13,25-27). The results of our study are in accordance with previous findings. The main advantage of this biomarker is that it is easily collected from routine blood tests before surgery, and thus, 
clinicians can conveniently tailor management strategies to individual patients. Third, this study first demonstrated that NAC toxicity is related to decreased OS and included this factor in the nomogram for OS prediction. In the nomogram, the predictive power of NAC toxicity appeared to be stronger than that of other factors. The results of our study suggest that when we evaluate the surgical risk of CRLM patients, it is important to focus on whether NAC toxicity occurs during the process of NAC treatment and to not ignore the condition of the patient during NAC. The reason for the unfavourable outcome in patients with NAC toxicity is largely unknown. One possible mechanism is that NAC toxicity represents damage to the body due to chemotherapy, especially the severe bone marrow suppression caused by chemotherapy, which is a signal of severe injury to the immune system (28). Combined with the sarcopenia caused by NAC toxicity $(29,30)$, NAC toxicity will further weaken anti-tumour capacity and lead to a poor prognosis. Fourth, this study stratified CRLM patients into high-risk, middle-risk and low-risk groups according to optimal threshold values, and a significant difference in PFS and OS was detected between the three risk groups. With regard to the different risk groups, clinicians might provide rational suggestions for additional individualized therapy and intensive follow-up. Last, our nomograms consisted of factors related to NAC conditions, preoperative test markers, surgical conditions and tumour biological characteristics, the applicable targets of which are relatively comprehensive. Improved nomogram accuracy often occurs at the cost of increased complexity, but our nomogram is concise, with only several predictive factors. In addition, all of the clinical factors in our nomograms are available during the perioperative period, without an additional burden to patients.

There were still several limitations in this study. First, this study was a typical single-institutional and retrospective study with a small sample size, and the nomograms were established with selection bias. Multi-institutional randomized control studies are necessary to further improve the sample size and reduce such selection bias. Second, the KRAS status was available for only $67.5 \%$ of the patients and was not incorporated into the present nomograms. Third, external validation from other institutions is needed to confirm the usefulness of these models. Although the models worked well in our internal cohort, multiinstitutional external validation would provide more convincing evidence.

In conclusion, in this study, upgraded nomograms specifically incorporating NAC-related factors (TRG, NAC toxicity) and preoperative testing markers (serum GGT level) for the prediction of postoperative complications, PFS, OS in CRLM patients undergoing NAC followed by resection were constructed, with favourable discrimination and calibration. The present nomograms will aid physicians in the early evaluation of the individual prognosis of patients and in the identification of high-risk patients who may need more aggressive treatment and follow-up strategies.

\section{Acknowledgments}

Funding: This study was supported by the State Key Project on Infection Diseases of China (grant No. 2017ZX10201021-007-003), the National The Capital Health Research and Development of Special (20181-4021), the National Natural Science Foundation of China (81672461, 81972311), CAMS Innovation Fund for Medical Sciences (CIFMS) (grant No. 2017-12M-4002), the Non-profit Central Research Institution Fund of Chinese Academy of Medical Sciences (2019PT310026) and Sanming Project of Medicine in Shenzhen (No. SZSM202011010).

\section{Footnote}

Reporting Checklist: The authors have completed the STROBE reporting checklist. Available at http://dx.doi. org/10.21037/atm-20-3973

Data Sharing Statement: Available at http://dx.doi. org/10.21037/atm-20-3973

Conflicts of Interest: All authors have completed the ICMJE uniform disclosure form (available at http://dx.doi. org/10.21037/atm-20-3973). The authors have no conflicts of interest to declare.

Ethics Statement: The authors are accountable for all aspects of the work in ensuring that questions related to the accuracy or integrity of any part of the work are appropriately investigated and resolved. The study conformed to the provisions of the Declaration of Helsinki (as revised in 2013). Ethical approval was obtained from our hospital (approval ID: NCC2019C-016). All participants gave informed consent before taking part in this study.

Open Access Statement: This is an Open Access article 
distributed in accordance with the Creative Commons Attribution-NonCommercial-NoDerivs 4.0 International License (CC BY-NC-ND 4.0), which permits the noncommercial replication and distribution of the article with the strict proviso that no changes or edits are made and the original work is properly cited (including links to both the formal publication through the relevant DOI and the license). See: https://creativecommons.org/licenses/by-nc-nd/4.0/.

\section{References}

1. Bray F, Ferlay J, Soerjomataram I, et al. Global cancer statistics 2018: GLOBOCAN estimates of incidence and mortality worldwide for 36 cancers in 185 countries. CA Cancer J Clin 2018;68:394-424.

2. Engstrand J, Nilsson H, Stromberg C, et al. Colorectal cancer liver metastases - a population-based study on incidence, management and survival. BMC Cancer 2018;18:78.

3. Benson AB 3rd, Venook AP, Cederquist L, et al. Colon Cancer, Version 1.2017, NCCN Clinical Practice Guidelines in Oncology. J Natl Compr Canc Netw 2017;15:370-98.

4. Diagnosis And Treatment Guidelines For Colorectal Cancer Working Group CSOCOC. Chinese Society of Clinical Oncology (CSCO) diagnosis and treatment guidelines for colorectal cancer 2018 (English version). Chin J Cancer Res 2019;31:117-34.

5. Sabanathan D, Eslick GD, Shannon J. Use of Neoadjuvant Chemotherapy Plus Molecular Targeted Therapy in Colorectal Liver Metastases: A Systematic Review and Meta-analysis. Clin Colorectal Cancer 2016;15:e141-e147.

6. Lim C, Doussot A, Osseis M, et al. Bevacizumab improves survival in patients with synchronous colorectal liver metastases provided the primary tumor is resected first. Clin Transl Oncol 2018;20:1274-9.

7. Imai K, Allard MA, Castro Benitez C, et al. Nomogram for prediction of prognosis in patients with initially unresectable colorectal liver metastases. Br J Surg 2016;103:590-9.

8. Liu W, Wang K, Han Y, et al. Nomogram predicted disease free survival for colorectal liver metastasis patients with preoperative chemotherapy followed by hepatic resection. Eur J Surg Oncol 2019;45:2070-7.

9. Serayssol C, Maulat C, Breibach F, et al. Predictive factors of histological response of colorectal liver metastases after neoadjuvant chemotherapy. World J Gastrointest Oncol 2019;11:295-309.
10. Chen Q, Wu C, Zhao H, et al. Neo-adjuvant Chemotherapy-Induced Neutropenia Is Associated with Histological Responses and Outcomes after the Resection of Colorectal Liver Metastases. J Gastrointest Surg 2020;24:659-70.

11. Okuno M, Hatano E, Seo S, et al. Indication for neoadjuvant chemotherapy in patients with colorectal liver metastases based on a nomogram that predicts disease-free survival. J Hepatobiliary Pancreat Sci 2014;21:881-8.

12. Stremitzer S, Stift J, Singh J, et al. Histological response, pattern of tumor destruction and clinical outcome after neoadjuvant chemotherapy including bevacizumab or cetuximab in patients undergoing liver resection for colorectal liver metastases. Eur J Surg Oncol 2015;41:868-74.

13. Chen Q, Zhao H, Wu J, et al. Preoperative D-dimer and Gamma-Glutamyltranspeptidase Predict Major Complications and Survival in Colorectal Liver Metastases Patients After Resection. Transl Oncol 2019;12:996-1004.

14. Rubbia-Brandt L, Giostra E, Brezault C, et al. Importance of histological tumor response assessment in predicting the outcome in patients with colorectal liver metastases treated with neo-adjuvant chemotherapy followed by liver surgery. Ann Oncol 2007;18:299-304.

15. Chen Q, Li C, Yang H, et al. Radiofrequency ablation versus resection for resectable liver metastases of gastrointestinal stromal tumours: Results from three national centres in China. Clin Res Hepatol Gastroenterol 2019;43:317-23.

16. Dindo D, Demartines N, Clavien PA. Classification of surgical complications: a new proposal with evaluation in a cohort of 6336 patients and results of a survey. Ann Surg 2004;240:205-13.

17. Wang H, Yang J, Yang J, et al. Development and validation of a prediction score for complications after hepatectomy in hepatitis B-related hepatocellular carcinoma patients. PLoS One 2014;9:e105114.

18. Liang Y, Lin C, Zhang B, et al. Perioperative outcomes comparing laparoscopic with open repeat liver resection for post-hepatectomy recurrent liver cancer: A systematic review and meta-analysis. Int J Surg 2020;79:17-28.

19. Hofmann FO, Holch JW, Heinemann V, et al. Prognostic value of radiologically enlarged lymph nodes in patients with metastatic colorectal cancer: Subgroup findings of the randomized, open-label FIRE-3/AIO KRK0306 trial. Eur J Radiol 2018;100:124-9.

20. Karachun A, Panaiotti L, Chernikovskiy I, et al. Shortterm outcomes of a multicentre randomized clinical 
Page 14 of 14

trial comparing D2 versus D3 lymph node dissection for colonic cancer (COLD trial). Br J Surg 2020;107:499-508.

21. Kim K, Kim YW, Shim H, et al. Differences in clinical features and oncologic outcomes between metastatic right and left colon cancer. J BUON 2018;23:11-8.

22. Shen H, Yang J, Huang Q, et al. Different treatment strategies and molecular features between right-sided and left-sided colon cancers. World J Gastroenterol 2015;21:6470-8.

23. Alsabilah J, Kim WR, Kim NK. Vascular Structures of the Right Colon: Incidence and Variations with Their Clinical Implications. Scand J Surg 2017;106:107-15.

24. Mao R, Zhao JJ, Bi XY, et al. A Low Neutrophil to Lymphocyte Ratio Before Preoperative Chemotherapy Predicts Good Outcomes After the Resection of Colorectal Liver Metastases. J Gastrointest Surg 2019;23:563-70.

25. Hofbauer SL, Stangl KI, de Martino M, et al. Pretherapeutic gamma-glutamyltransferase is an independent prognostic factor for patients with renal cell carcinoma. Br J Cancer 2014;111:1526-31.

Cite this article as: Chen Q, Mao R, Zhao J, Bi X, Li Z, Huang Z, Zhang Y, Zhou J, Zhao H, Cai J. Upgraded nomograms for the prediction of complications and survival in patients with colorectal liver metastases treated with neoadjuvant chemotherapy followed by hepatic resection. Ann Transl Med 2021;9(3):265. doi: 10.21037/atm-20-3973

\section{Chen et al. Nomograms for the prediction of prognosis in CRLM}

26. Ma H, Zhang L, Tang B, et al. gammaGlutamyltranspeptidase is a prognostic marker of survival and recurrence in radiofrequency-ablation treatment of hepatocellular carcinoma. Ann Surg Oncol 2014;21:3084-9.

27. Yang F, Zhang S, Yang H, et al. Prognostic significance of gamma-glutamyltransferase in patients with resectable esophageal squamous cell carcinoma. Dis Esophagus 2015;28:496-504.

28. Family L, Li Y, Chen LH, et al. A Study of Novel Febrile Neutropenia Risk Factors Related to Bone Marrow or Immune Suppression, Barrier Function, and Bacterial Flora. J Natl Compr Canc Netw 2018;16:1201-8.

29. Barret M, Antoun S, Dalban C, et al. Sarcopenia is linked to treatment toxicity in patients with metastatic colorectal cancer. Nutr Cancer 2014;66:583-9.

30. Robinson S, Granic A, Sayer AA. Nutrition and Muscle Strength, As the Key Component of Sarcopenia: An Overview of Current Evidence. Nutrients 2019;11:2942. 